\title{
The difficulty of inferring progenitor masses from type-Il-Plateau supernova light curves
}

\author{
Luc Dessart $^{1}$ and D. John Hillier ${ }^{2}$ \\ 1 Unidad Mixta Internacional Franco-Chilena de Astronomía (CNRS, UMI 3386), Departamento de Astronomía, \\ Universidad de Chile, Camino El Observatorio 1515, Las Condes, Santiago, Chile \\ e-mail: Luc.Dessart@oca.eu \\ 2 Department of Physics and Astronomy \& Pittsburgh Particle Physics, Astrophysics, and Cosmology Center (PITT PACC), \\ University of Pittsburgh, 3941 O'Hara Street, Pittsburgh, PA 15260, USA
}

Received 28 November 2018 / Accepted 8 March 2019

\begin{abstract}
Much controversy surrounds the inferred progenitor masses of type-II-Plateau (II-P) supernovae (SNe). The debate is nourished by the discrepant results from radiation-hydrodynamics simulations, pre-explosion imaging, and studies of host stellar populations. Here, we present a controlled experiment using four solar-metallicity models with zero-age main sequence masses of $12,15,20$, and $25 M_{\odot}$. Because of the effects of core burning and surface mass loss, these models reach core collapse as red-supergiant (RSG) stars with a similar H-rich envelope mass of 8 to $9 M_{\odot}$ but with final masses in the range 11 to $16 M_{\odot}$. We explode the progenitors using a thermal bomb, adjusting the energy deposition to yield an asymptotic ejecta kinetic energy of $1.25 \times 10^{51} \mathrm{erg}$ and an initial ${ }^{56} \mathrm{Ni}$ mass of $0.04 M_{\odot}$. The resulting SNe produce similar photometric and spectroscopic properties from 10 to $200 \mathrm{~d}$. The spectral characteristics are degenerate. The scatter in early-time color results from the range in progenitor radii, while the differences in latetime spectra reflect the larger oxygen yields in more massive progenitors. Because the progenitors have a comparable H-rich envelope mass, the photospheric phase duration is comparable for all models; the difference in He-core mass is invisible. As different main sequence masses can produce progenitors with a similar H-rich envelope mass, light-curve modeling cannot provide a robust and unique solution for the ejecta mass of type-II-P SNe. The numerous uncertainties in massive-star evolution and wind-mass loss also prevent a robust association with a main sequence star mass. Light-curve modeling can at best propose compatibility.
\end{abstract}

Key words. radiative transfer - radiation: dynamics - supernovae: general

\section{Introduction}

Understanding how the landscape of type-II-Plateau (II-P) supernova (SN) properties connects to the diversity of redsupergiant (RSG) star progenitors and their explosion is of great interest for astrophysics. Unfortunately discrepant estimates of the ejecta and progenitor masses are often obtained from radiation-hydrodynamics simulations that study the bolometric light-curve evolution ${ }^{1}$, from pre-explosion imaging, and from studies of host stellar populations.

While significant advances have been made in the modeling of the proto-neutron star phase leading to shock revival and explosion in massive star progenitors, there are still many unresolved issues. Although the ejecta properties in these studies are broadly consistent with inferred properties from type-II-P SN observations (Lentz et al. 2015; Müller et al. 2017; Glas et al. 2019; O'Connor \& Couch 2018; Vartanyan et al. 2019), the simulations cannot predict which stars produce core-collapse $\mathrm{SNe}$, or quantities such as the ${ }^{56} \mathrm{Ni}$ mass and explosion energy. Complications also arise from uncertainties in the structure of the SN progenitor prior to core collapse. These theoretical studies would benefit from reliable observational inferences about SN and progenitor properties.

\footnotetext{
1 We refer loosely to this approach as "light-curve modeling". In practice, it usually (but not always) includes the additional information from one spectral line to infer the evolution of the expansion rate at the photosphere.
}

Using pre-explosion photometry, observations have allowed the detection of a progenitor RSG for a handful of type-II-P SNe (Van Dyk et al. 2003, 2012a,b; Smartt et al. 2004; Maund et al. 2005, 2013, 2014; Fraser et al. 2012, 2014; O’Neill et al. 2019). These works propose main sequence progenitor masses below about $17 M_{\odot}$ (and the lack of more massive RSG progenitors has been coined the "RSG" problem), although these inferences are often uncertain. The problem of inferring the progenitor luminosity is made difficult for example because of the frequent lack of multi-band photometry, the uncertain bolometric correction (Davies \& Beasor 2018), or the uncertain reddening (Beasor \& Davies 2016). Furthermore, these inferences are based on stellar evolution models, whose predictions depend on physical properties that are either not known a priori (e.g., initial rotation) or are hard to model (e.g., convection, overshooting; Arnett et al. 2015). These can impact the He-core mass and the progenitor luminosity at the time of explosion (Heger et al. 2000; Meynet \& Maeder 2000; Hirschi et al. 2004).

Radiation hydrodynamics and radiative transfer can also be used to characterize the properties of type-II-P SNe. The most robust inference and the most easily done is for the ${ }^{56} \mathrm{Ni}$ mass since full $\gamma$-ray trapping holds at the onset of the nebular phase. At that time, the bolometric luminosity equals the total power radiated from ${ }^{56} \mathrm{Ni}$ and ${ }^{56} \mathrm{Co}$ decay, and the mass of ejected ${ }^{56} \mathrm{Ni}$ can be determined at an accuracy that depends only on the accuracy of the adopted distance, reddening, and amount of flux falling outside of the observed range (e.g., Woosley 1988; 
Hamuy 2003). Other inferences such as explosion energy, ejecta mass, and progenitor mass at collapse or on the main sequence require some modeling and are therefore more uncertain.

Radiation hydrodynamic simulations of type-II-P SNe have been performed for several decades. Early works identified the basic properties of the progenitors, in particular their large progenitor radii and massive $\mathrm{H}$-rich envelopes compatible with RSG progenitors (Grassberg et al. 1971; Falk \& Arnett 1977). Basic relations between ejecta and observed type-II-P SN properties were also drawn (Litvinova \& Nadezhin 1985; Popov 1993). When applied to observed type-II-P SNe, these relations can however produce very perplexing numbers, such as ejecta masses of several $10 M_{\odot}$ or small supergiant radii atypical of RSG stars (see, e.g., Hamuy 2003). In many cases, these quantities conflict with predictions from stellar evolution and progenitor observations.

Studies dedicated to specific objects allow for a more refined analysis through the production of a tailored model (see, e.g., Turatto et al. 1998; Utrobin 2007; Chugai \& Utrobin 2000; Bersten et al. 2011; Dessart et al. 2013; Lisakov et al. 2018; Morozova et al. 2018). But in this case, one notes a large disparity in inferred ejecta properties, in particular the ejecta mass, which then translates into an even larger disparity in the progenitor main sequence mass. These masses are often much larger than those inferred for the progenitor stars from pre-explosion imaging (Smartt 2009), or from the modeling of nebular-phase spectra (Maguire et al. 2012; Jerkstrand et al. 2012, 2014). Since mass is the most defining characteristic of a star, this discrepancy is a problem.

Type-II-Plateau SN light curves are however primarily sensitive to the H-rich envelope mass and not to the mass of the progenitor He-core (Dessart \& Hillier 2011a; Dessart et al. 2013). Therefore, in many instances the inferred "ejecta" mass refers only to the H-rich envelope mass of the progenitor star at the time of explosion. This ambiguity arises because the words envelope mass and ejecta mass are used interchangeably in the literature, while the two words refer to two different entities. Stellar evolution indicates that their value may differ by a factor of ten.

To clarify this property, we use the public grid of massive star models from Woosley et al. (2002). Figure 1 shows a montage of density profiles at core collapse for massive stars evolved at solar metallicity with zero-age main sequence masses ( $M_{\text {ZAMS }}$ ) of 11 up to $30 M_{\odot}$. The left column shows the standard way of presenting such profiles, with the origin at the center of the star. As the initial mass $M_{\mathrm{ZAMS}}$ increases, the mass of the He core $\left(M_{\mathrm{He}, \mathrm{c}}\right.$; shown in red, and corresponding to regions with a density greater than about $1 \mathrm{~g} \mathrm{~cm}^{-3}$ ) increases from about $3 M_{\odot}$ for the $11 M_{\odot}$ model up to $10 M_{\odot}$ for the $30 M_{\odot}$ model. It is in this hot and dense $\mathrm{He}$ core that nuclear fusion takes place. In the lowerdensity lower-temperature H-rich envelope, the composition deviates from its primordial value only through the effect of mixing (see, e.g., Davies \& Dessart 2019). The mass of the H-rich envelope $\left(M_{\mathrm{H}, \mathrm{e}}\right)$ at core collapse is between 8 and $9 M_{\odot}$ for stars with $M_{\text {ZAMS }}$ between 11 and $21 M_{\odot}$, and decreases (not necessarily monotonically) as $M_{\text {ZAMS }}$ increases further. This arises from an empirically based formulation of RSG mass-loss rates, which reflects the greater mass-loss rates inferred for more massive and more luminous RSGs (de Jager et al. 1988). This influence of mass loss on $M_{\mathrm{H}, \mathrm{e}}$ is what eventually turns more massive stars into H-deficient Wolf-Rayet stars (Maeder \& Meynet 1987; Langer et al. 1994; Crowther 2007). While RSG star mass loss remains uncertain, this should only affect the value of $M_{\text {ZAMS }}$ where $M_{\mathrm{H}, \mathrm{e}}$ starts decreasing with increasing $M_{\mathrm{ZAMS}}$, and the actual value of $M_{\mathrm{H}, \mathrm{e}}$ for a given $M_{\mathrm{ZAMS}}{ }^{2}$. The trend shown in Fig. 1 must hold.

Because type-II-P SN light curves are primarily sensitive to the H-rich envelope mass, it is more instructive to show the density structure with respect to the edge of the He core (right column of Fig. 1). Ignoring the $\mathrm{He}$ core, the models with $M_{\text {ZAMS }}$ below $21 M_{\odot}$ have similar density structures, while above the models have progressively lower values of $M_{\mathrm{H}, \mathrm{e}}$. For a given ejecta kinetic energy $E_{\text {kin }}$ imparted to the H-rich envelope, models with $M_{\text {ZAMS }}$ below $21 M_{\odot}$ should have comparable light curves (modulo differences in $R_{\star}$ ), while above $21 M_{\odot}$, the light curve should be characterized by a faster postbreakout luminosity decline and a shorter photospheric phase (Bartunov \& Blinnikov 1992). Furthermore, "ejecta" masses (i.e., $M_{\mathrm{H}, \mathrm{e}}$ values) inferred from type-II-P SN light curves should consequently be around $10 M_{\odot}$ since the RSG stars from 10-20 $M_{\odot}$ stars on the main sequence are favored by the initial mass function. Masses of just a few $M_{\odot}$ (which result from higher-mass progenitors) should be less frequently inferred (the reasoning here ignores binarity). This basic picture seems compatible with the observed diversity of type-II SN light curves (Anderson et al. 2014; Sanders et al. 2015). Furthermore, the large range of type-II SN ejecta masses (extending to large masses) published in the literature appears incongruous.

The goal of this paper is to demonstrate that the type-II-P SN ejecta masses inferred from light-curve modeling bear considerable uncertainty. One cannot infer the progenitor mass (either at core collapse or on the main sequence) using light-curve modeling. The problem is degeneracy since stars with widely different total masses may have the same $M_{\mathrm{H}, \mathrm{e}}$ at core collapse. To illustrate this property, we conduct a controlled experiment on a set of massive star models with different main sequence mass but the same H-rich envelope mass - our model set is very similar to that of Woosley et al. (2002) and thus representative of predictions for single star evolution. The next section presents the numerical approach. Section 3 then presents the $\mathrm{SN}$ radiative properties for each model and discusses the implications of the results.

\section{Numerical approach}

The progenitor models correspond to solar-metallicity nonrotating stars of $12,15,20$, and $25 M_{\odot}$ on the main sequence. These were evolved with MESA version 7623 (Paxton et al. 2011, 2013, 2015) as part of earlier works (Lisakov et al. 2017, 2018). These stars are evolved as single stars, or in binary systems with a wide orbit: the possibility of mass exchange with a companion star is ignored. These simulations use standard MESA default parameters, except for a mixing length parameter of three. This choice is needed to produce more compact RSG stars at core collapse (see Dessart et al. 2013, and more recently Paxton et al. 2018). Because this produces RSG star models with a higher effective temperature, a slight enhancement on the default massloss rate parameter is needed in order to yield a mass-loss rate similar to that of RSGs with a lower effective temperature. The model properties are summarized in Table 1, while Fig. 2 shows the density structure at the onset of core collapse for each model. The models differ in He-core mass but have a similar H-rich envelope mass - their properties are similar to those of Woosley et al. (2002) for the corresponding ZAMS mass (Fig. 1). In radial space, the differences in He core properties are invisible since the He core has the size of the Sun while the surface radii cover approximately from 400 to $900 R_{\odot}$.

2 These quantities should also vary with metallicity. 

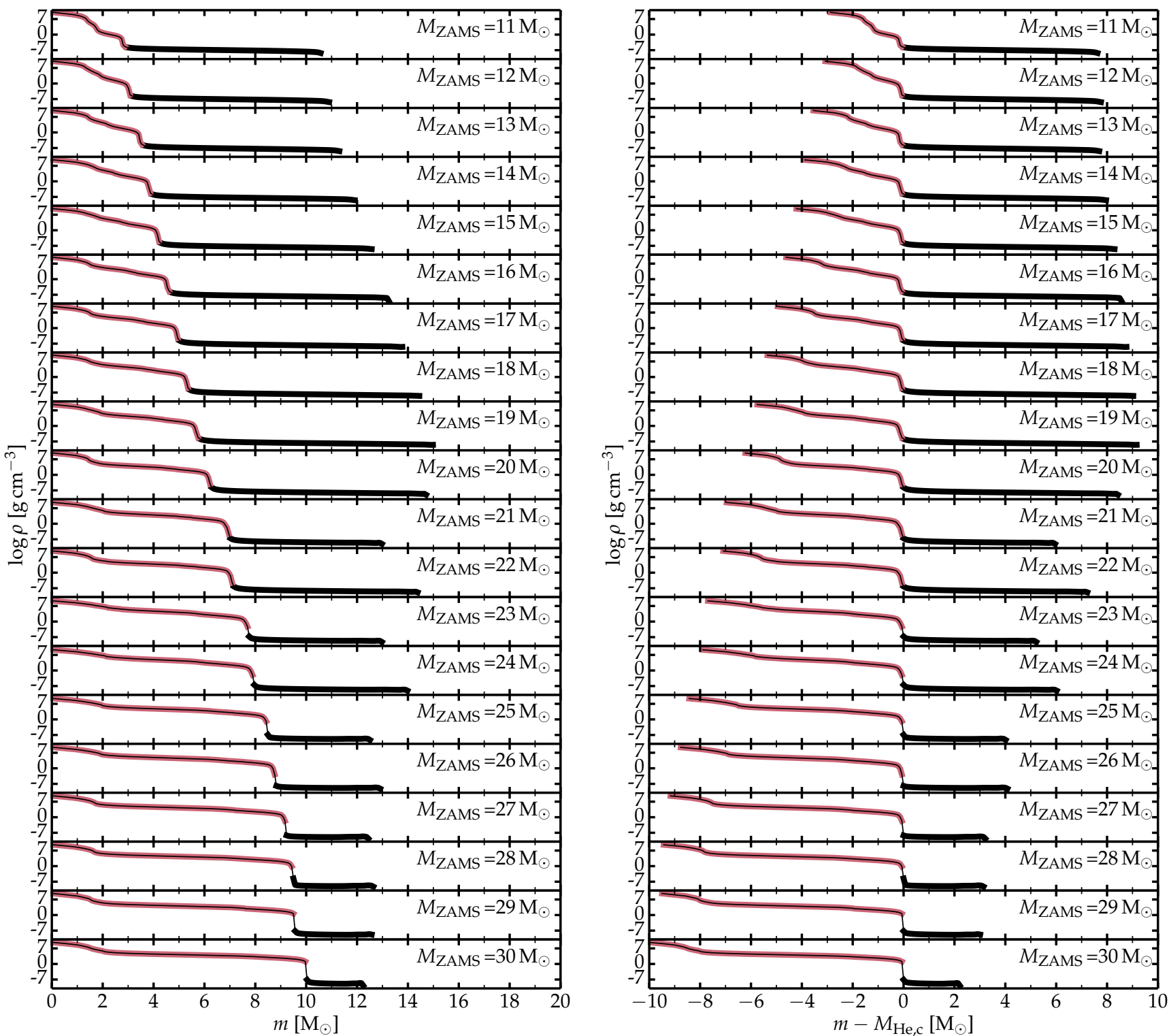

Fig. 1. Density profiles vs. Lagrangian mass at the time of core collapse for a set of stellar models with an initial mass on the main sequence between 11 and $30 M_{\odot}$ (Woosley et al. 2002). The origin is at star center (left column) or at the He core edge (right column). The H-rich envelope is drawn with a thick black line and corresponds in all models to (extended) regions with a density below about $10^{-7} \mathrm{~g} \mathrm{~cm}^{-3}$. The He core is drawn with a thick red line and corresponds in all models to (compact) regions with a density above about $1 \mathrm{~g} \mathrm{~cm}^{-3}$. The vertical scale is squeezed but is suitable to reveal the evolution of the He core mass and the H-rich envelope mass for different ZAMS masses. Left panel: smooth progression in the $\mathrm{He}$ core mass with initial progenitor mass, and the varying progenitor mass at the onset of core collapse. Right panel: nonlinear evolution of the H-rich envelope mass with initial progenitor mass is emphasized. In these models, stars with ZAMS masses of 11-21 $M_{\odot}$ contain similar H-rich envelope masses (8-9 $M_{\odot}$ ) at the onset of core collapse.

Because they make no distinction between the high-density He core and the low-density H-rich envelope, polytropic progenitor structures are unsuitable for light-curve modeling. To comply with the early-time observations of some type-II-P SNe (see, e.g., Yaron et al. 2017), about $\sim 0.2 M_{\odot}$ of atmospheric (static) material is added above $R_{\star}$ using a density scale height of about $0.2 R_{\star}$. This has however no relevance for the present discussion.

The explosion is modeled with the 1D gray radiationhydrodynamics code v1D in the usual manner (Livne 1993; Dessart et al. 2010). The explosion is triggered by means of a piston placed within the Si-rich shell, at a mass cut of 1.5$1.94 M_{\odot}$. To compensate for the progenitor binding energy, the explosion energy is adjusted for each model to yield an asymptotic ejecta kinetic energy $E_{\text {kin }}$ of $\sim 1.25 \times 10^{51} \mathrm{erg}$. Although
v1D treats explosive nucleosynthesis, the ${ }^{56} \mathrm{Ni}$ mass is reset to be $0.04 M_{\odot}$ at $100 \mathrm{~s}$ after the piston trigger (the value in Table 1 deviates a little because of the remapping into CMFGEN). At $14 \mathrm{~d}$ after the piston trigger (when we remap into CMFGEN), the four models have essentially the same density structure in velocity space all the way down to the He-core material (whose outer location corresponds to the density jump; Fig. 3). The jump is at a velocity of $1000-2000 \mathrm{~km} \mathrm{~s}^{-1}$ greater in model $\mathrm{m} 25$ than in model $\mathrm{m} 12$, which reflects the differences in He-core mass between the models (see Dessart et al. 2010 for discussion). The temperature above that jump is lower in more compact progenitor models. The material that used to be in the He core has cooled considerably because it expanded by a factor of about 1000 , while the H-rich envelope has expanded by a factor of about ten. 
Table 1. Properties of the model set.

\begin{tabular}{ccccccccc}
\hline \hline & $\begin{array}{c}M_{\mathrm{fin}} \\
\left(M_{\odot}\right)\end{array}$ & $\begin{array}{c}M_{\mathrm{He}, \mathrm{c}} \\
\left(M_{\odot}\right)\end{array}$ & $\begin{array}{c}M_{\mathrm{H}, \mathrm{e}} \\
\left(M_{\odot}\right)\end{array}$ & $\begin{array}{c}R_{\star} \\
\left(R_{\odot}\right)\end{array}$ & $\begin{array}{c}E_{\mathrm{kin}} \\
(\mathrm{erg})\end{array}$ & $\begin{array}{c}M_{\mathrm{ej}} \\
\left(M_{\odot}\right)\end{array}$ & $\begin{array}{c}M(\mathrm{O}) \\
\left(M_{\odot}\right)\end{array}$ & $\begin{array}{c}M\left({ }^{56} \mathrm{Ni}\right) \\
\left(M_{\odot}\right)\end{array}$ \\
\hline $\mathrm{m} 12$ & 11.21 & 3.26 & 7.95 & 406 & $1.27(51)$ & 9.79 & 0.16 & 0.046 \\
$\mathrm{~m} 15$ & 13.12 & 4.56 & 8.56 & 589 & $1.28(51)$ & 11.57 & 0.53 & 0.041 \\
$\mathrm{~m} 20$ & 14.86 & 6.81 & 8.05 & 843 & $1.25(51)$ & 13.18 & 1.33 & 0.041 \\
$\mathrm{~m} 25$ & 15.96 & 8.59 & 7.37 & 872 & $1.21(51)$ & 13.93 & 2.33 & 0.042 \\
\hline
\end{tabular}

Notes. The table includes the final mass at core collapse $\left(M_{\mathrm{fin}}\right)$, the Hecore mass $\left(M_{\mathrm{He}, \mathrm{c}}\right)$, the $\mathrm{H}$-rich envelope mass $\left(M_{\mathrm{H}, \mathrm{e}}\right)$, the surface radius $\left(R_{\star}\right)$, the ejecta kinetic energy $\left(E_{\mathrm{kin}}\right)$, the ejecta mass $\left(M_{\mathrm{ej}}\right)$, the oxygen mass $(M(\mathrm{O}))$, and the ${ }^{56} \mathrm{Ni}$ mass. The initial mass on the main sequence is reflected in the model name, for example $12 M_{\odot}$ for model $\mathrm{m} 12$. Numbers in parenthesis represent powers of ten.

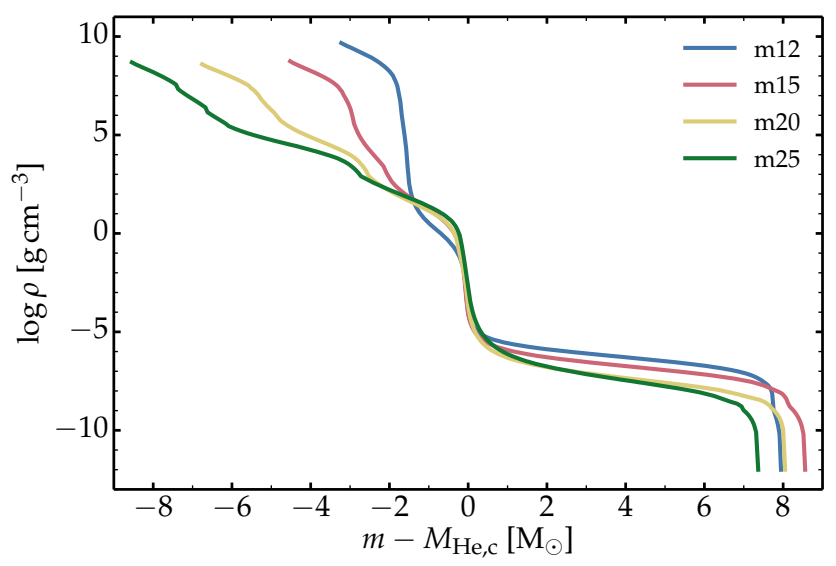

Fig. 2. Density structure of the models $\mathrm{m} 12$, $\mathrm{m} 15$, $\mathrm{m} 20$, and $\mathrm{m} 25$ shown vs. Lagrangian mass (the origin is the outer edge of the He core). The differences in H-rich envelope density reflect the differences in surface radii (see Table 1).

This conspires to make the temperature very uniform throughout most of the ejecta. The relatively small volume occupied by the former He core stores a modest radiative energy. Instead, the large volume occupied by the shocked H-rich envelope stores most of the radiative energy that will be released during the highbrightness phase of a type-II-P SN. It also stores the bulk of the kinetic energy.

At $14 \mathrm{~d}$, the ejecta models computed by v1D are remapped into the nonlocal thermodynamic equilibrium (nonLTE) and time-dependent radiative-transfer code CMFGEN (Hillier \& Dessart 2012). The evolution is then followed until $300 \mathrm{~d}$ using the standard procedure (see, e.g., Dessart et al. 2013).

There is at present no robust theory that predicts with certainty the ejecta kinetic energy and ${ }^{56} \mathrm{Ni}$ mass that a given ZAMS mass should produce. However, the simulations of Sukhbold et al. (2016) suggest that stars in a broad mass range from about 12 to $25 M_{\odot}$ can yield an ejecta within $50 \%$ of $1.2 \times 10^{51} \mathrm{erg}$ and a ${ }^{56} \mathrm{Ni}$ mass within a factor of two of $0.04 M_{\odot}$ (their is some scatter and some offset with $M_{\text {ZAMS }}$ depending on the calibration used for their "thermal bomb"). Our choice is therefore realistic.

\section{Results and discussion}

Figure 4 summarizes the main results from the CMFGEN simulations for models $\mathrm{m} 12, \mathrm{~m} 15, \mathrm{~m} 20$, and $\mathrm{m} 25$. The bolometric light curves are similar. Models with larger progenitor radii are more
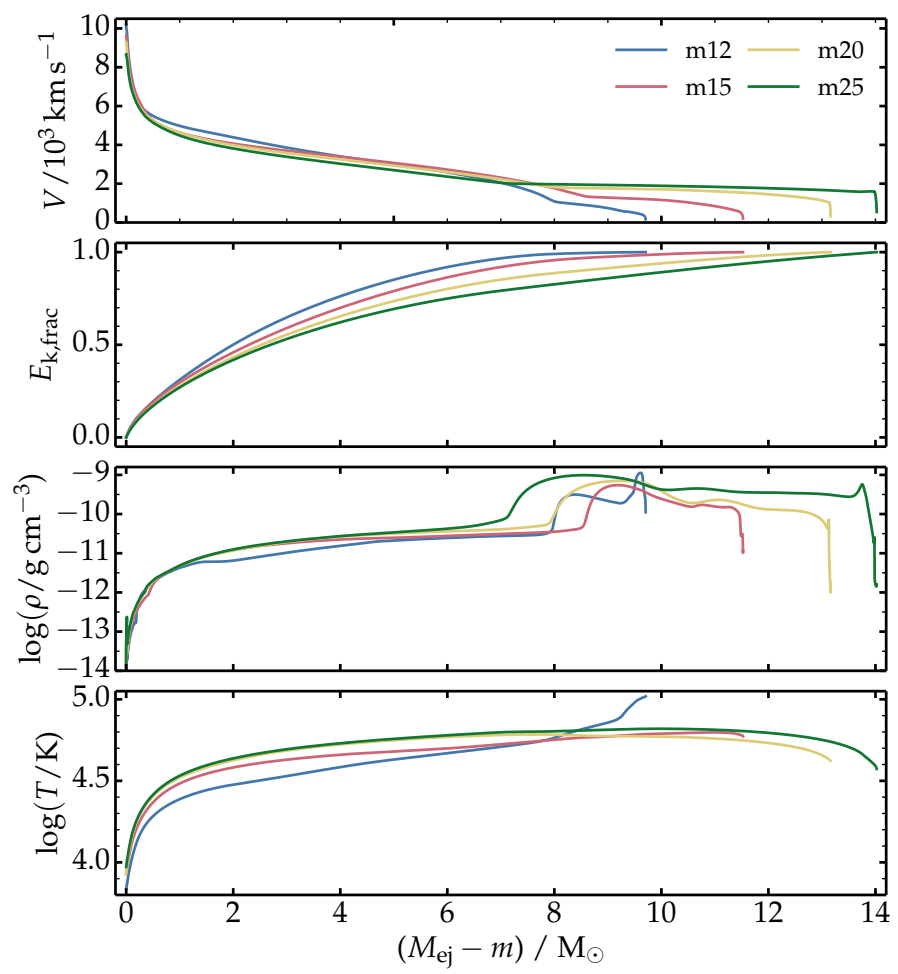

Fig. 3. Ejecta velocity, fractional kinetic energy $E_{\mathrm{k}, \text { frac }}$, density, and temperature vs. mass at $14 \mathrm{~d}$ after the piston trigger. $E_{\mathrm{k}, \text { frac }}$ is defined as $\int V^{2} \mathrm{dm} / 2 E_{\text {kin }}$, with the integration done inward. The $\mathrm{x}$-axis origin is at the outermost ejecta layer in order to emphasize how similar the properties of the shocked H-rich envelope are in these different progenitor models.

luminous, as expected (see., e.g., Popov 1993; Kasen \& Woosley 2009; Dessart et al. 2013). The $V$-band light curve and the $V-I$ color evolution are also similar, with the bigger progenitors reddening later and being visually brighter (this is the same as for models s15 and s25 in Dessart \& Hillier 2011a and for models m15mlt1 and m15mlt3 in Dessart et al. 2013). These color offsets are understood from the offset in photospheric temperature (also visible in the $\mathrm{H}$ ionization state), with the bigger progenitors remaining hotter at the photosphere for longer. Despite the large differences in progenitor and ejecta masses, the four models have a similar photospheric-phase duration because they have a similar H-rich envelope mass (Fig. 2). The models show the same qualitative and quantitative evolution in photospheric velocity, because the bulk of the kinetic energy is held up in what used to be the H-rich envelope. Throughout the photospheric phase, the $\mathrm{H}$ mass fraction at the photosphere is comparable in all models (i.e., the photosphere resides in the former H-rich envelope; Dessart \& Hillier 2011a) which implies that the spectra should reveal lines from similar elements.

While the light curves and colors show some differences, these are not sufficient to unambiguously determine the ejecta mass. The main driver behind the differences in photometric properties is the progenitor radius, not the ejecta mass. If we had used a different mixing length parameter for the four progenitor models (smaller in lower mass models), we could have produced progenitors with the same radius, and in that case the light curves would have been even more similar. Furthermore, because RSG observations probe the outer low-density fluff of the RSG atmosphere rather than the higher-density hydrostatic photosphere (as defined in stellar evolution models), surface radii are poorly constrained. Further, and contrary to a generally held belief, RSG 

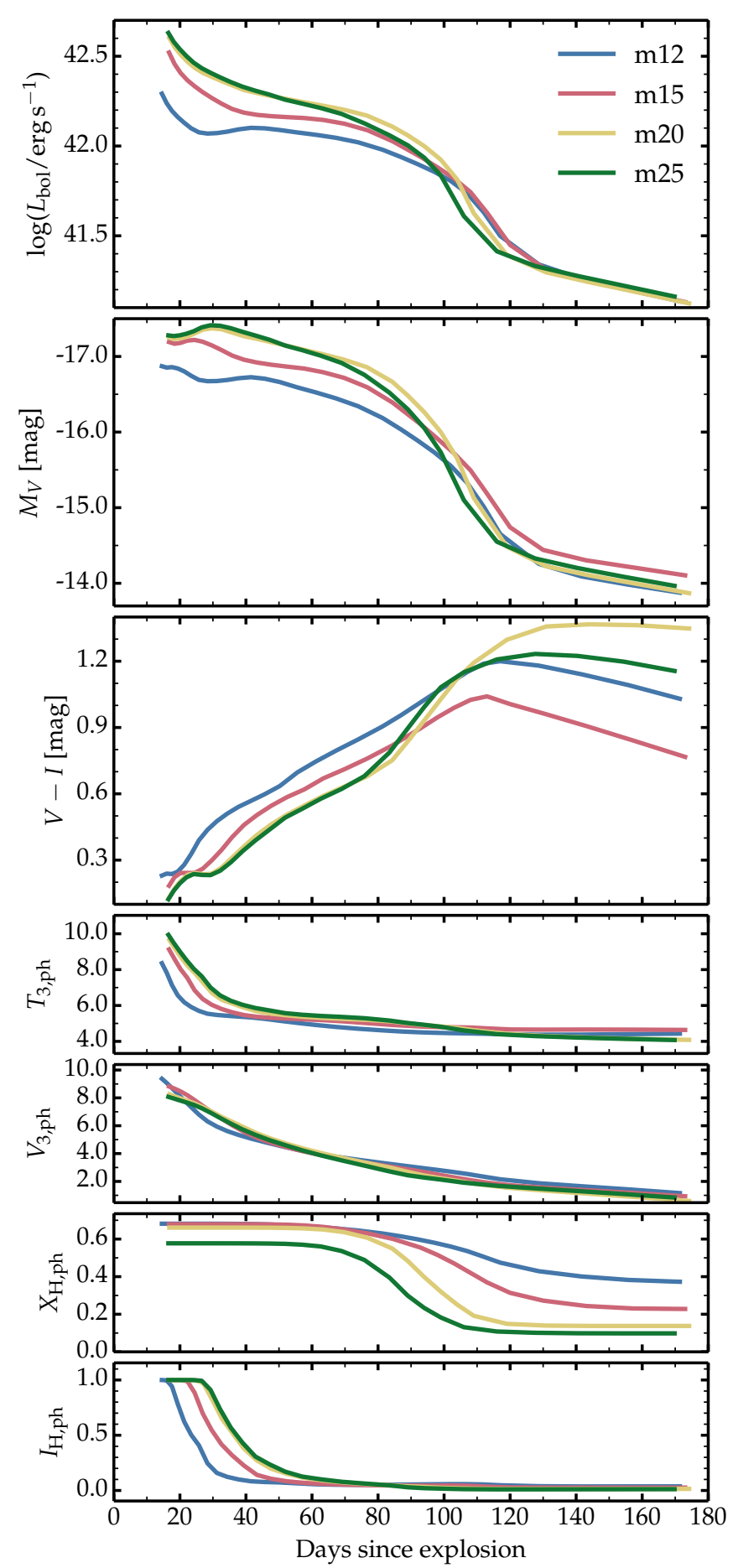

Fig. 4. Summary of results from the CMFGEN simulations of models $\mathrm{m} 12$, $\mathrm{m} 15, \mathrm{~m} 20$, and $\mathrm{m} 25$, showing, from top to bottom, the bolometric light curve, the $V$-band light curve, the $V-I$ color evolution, and the evolution of some photospheric properties $\left(T_{3, \mathrm{ph}}\right.$ : temperature in units of $1000 \mathrm{~K}$; $V_{3, \mathrm{ph}}$ : velocity in units of $1000 \mathrm{~km} \mathrm{~s}^{-1} ; X_{\mathrm{H}, \mathrm{ph}}$ : hydrogen mass fraction; $I_{\mathrm{H}, \mathrm{ph}}:$ hydrogen ionization state).

radii are not predicted with any confidence by stellar-evolution models since $R_{\star}$ is controlled by convection and a prescribed mixing length (Maeder \& Meynet 1987; Dessart et al. 2013).

Other factors also influence the light curves. For example, there is increasing evidence that the early-time light curves of many type-II-P SNe are influenced by circumstellar (CSM) interaction, which affects both the brightness and colors of the SN at early times (e.g., Yaron et al. 2017; Morozova et al. 2017, 2018; Dessart et al. 2017; Moriya et al. 2017; Förster et al. 2018). Clumping may also hasten the photosphere recession and boost the SN luminosity during the high-brightness phase (Dessart et al. 2018). Further, most simulations of core-collapse $\mathrm{SNe}$ lead to asymmetric explosions. This may affect both the light-curve evolution and colors. Observations of type-II-P SNe also show variable levels of polarization, and the degree of polarization is time-dependent (Leonard et al. 2006, 2012). The latter may indicate (although not necessarily) that the degree of asymmetry varies with time (Dessart \& Hillier 2011b). For example, the core may be much more asymmetric than the massive H-rich envelope. The amount of ${ }^{56} \mathrm{Ni}$ mixing is also known to affect the shape of the light curve (see, e.g., Kasen \& Woosley 2009), and there are observational issues related to the distance of the $\mathrm{SN}$, reddening and the inference of the bolometric light curve from a finite number of bands. Finally, we stress that even if one can infer the ejecta mass from the light curve, an extrapolation to the progenitor mass is fraught with biases because RSG massloss rates, and hence the progenitor mass-loss history, are poorly known (see, e.g., Fig. 1 in Meynet et al. 2015).

In nature, it is possible that the explosion of progenitors with very different ZAMS masses yields the same asymptotic ejecta kinetic energy (which is what we assumed here for our model set) and therefore naturally contributes to light-curve degeneracy. The power source for the explosion is gravitational contraction of the core and mass accretion onto it (see, e.g., Burrows \& Goshy 1993). This process may release more energy in higher-mass progenitors, but this additional energy would be sapped by the greater binding energy of their He core. In the present set of models, to produce an ejecta kinetic energy of $1.25 \times 10^{51} \mathrm{erg}$, the total energy released by the "thermal bomb" had to be larger in more massive progenitors. Specifically, it was $1.36(\mathrm{~m} 12), 1.39(\mathrm{~m} 15), 1.78(\mathrm{~m} 20)$, and $1.96 \times 10^{51} \mathrm{erg}(\mathrm{m} 25)-$ these values depend on the time left before the onset of collapse (e.g., the central density is not the same in all models). The trend nonetheless holds (see, e.g., Woosley et al. 2002).

Polytropes and nonevolutionary progenitor structures, designed with considerable freedom, conflict with the fundamental features of massive stars at death (primarily through the incorrect treatment of their core-halo structure). It is from these simulations that the largest discrepancies in progenitor masses arise (see also Sect. 4.1 in Morozova et al. 2018). Similarly, analytic scalings (e.g., Litvinova \& Nadezhin 1985) are unable to yield a reliable ejecta mass since they are insensitive to the $\mathrm{He}$ core mass.

An extensive discussion about progenitor masses is provided by Dall'Ora et al. (2014). In order to examine the parameter space they considered both a semi-analytic approach and an approach based on direct hydrodynamical modeling. Their semianalytic approach yields multiple minima in $\chi^{2}$ when comparing model predictions with observations. While not all solutions gave consistency with the inferred photospheric velocities, these studies do highlight degeneracies in inferring ejecta masses from light curves.

Morozova et al. (2018) have also performed a parameter study of type-II-P light curves. Unlike the study of Dall'Ora et al. (2014), their simulations are based on evolutionary models computed with KEPLER. With some exceptions, the $2 \mathrm{D}-\chi^{2}$ plots of Morozova et al. (2018) generally show a broad range of ZAMS masses and radii that are consistent with their light curves. Given the degeneracies, assumptions in both the evolutionary models and in the light-curve modeling will significantly influence the results. Furthermore, Morozova et al. (2018) 

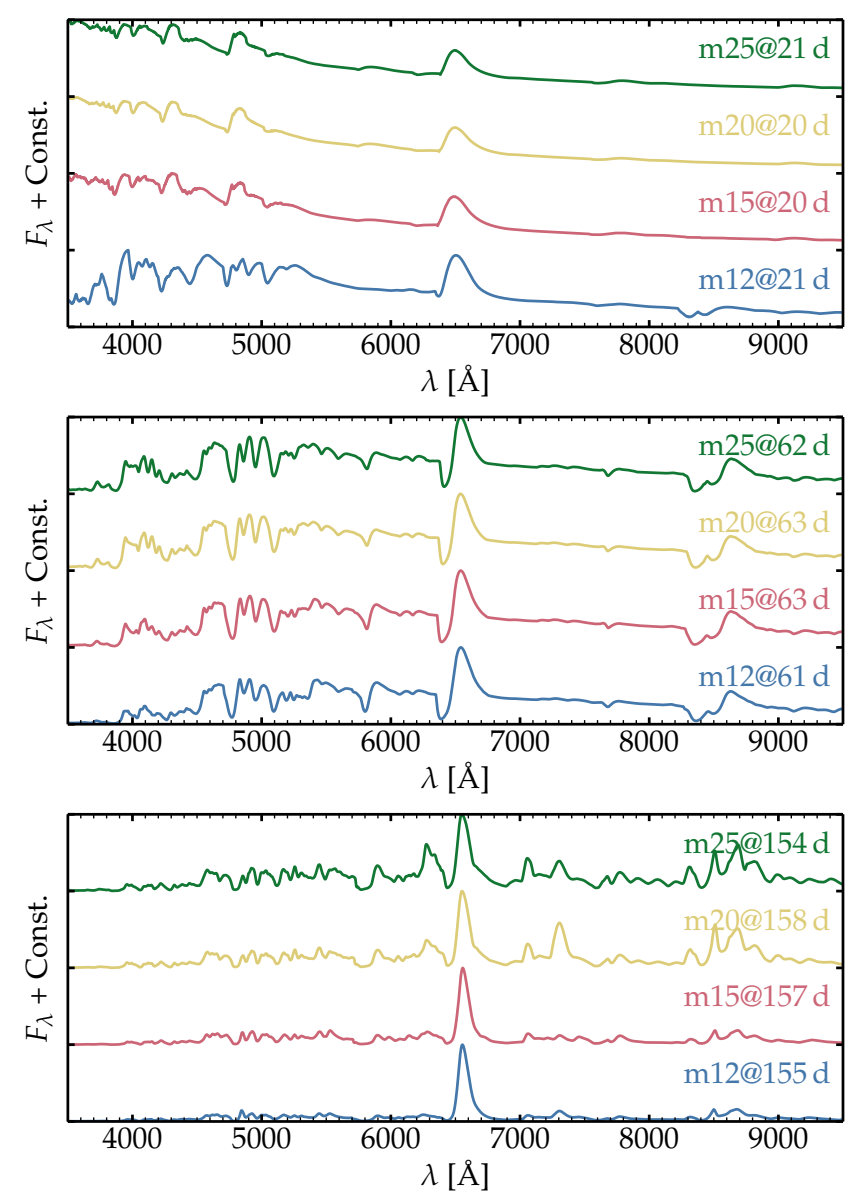

Fig. 5. Comparison of optical spectra for models $\mathrm{m} 12, \mathrm{~m} 15, \mathrm{~m} 20$, and $\mathrm{m} 25$ at about $20 \mathrm{~d}$ (top), $62 \mathrm{~d}$ (middle), and $155 \mathrm{~d}$ (bottom) after explosion. The color offset at early times results from the different progenitor radii. The greater O $16300 \AA$ line in more massive progenitors arises from their greater oxygen content.

infer explosion energies and ejecta masses without using any spectral information, hence ignoring fundamental constraints on the expansion rate. For example, their ejecta kinetic energies are lower than standard, and with their adopted CSM mass, their ejecta models most likely fail to produce the broad Dopplerbroadened lines seen in type-II SN spectra. Disregarding spectral constraints, light-curve modeling is even more subject to degeneracies.

While more difficult to model, spectra potentially provide another avenue to constrain ejecta and progenitor mass. Figure 5 shows the spectra for our model set at about 20,62, and $155 \mathrm{~d}$ after explosion. At the first epoch, the difference is the greatest and results from the larger radii in more massive progenitors (which cause the brightness and color offset shown in Fig. 4). However, during the recombination phase (second epoch), the spectra are essentially identical. At the nebular epoch, the spectra are also very similar; they exhibit a strong $\mathrm{H} \alpha$ line, but with a stronger O I $6300 \AA$ doublet line in more massive progenitors.

A more reliable discriminant for progenitor mass may be sought from nebular-phase spectra. At such times, the stark contrast between a 12 and a $25 M_{\odot}$ progenitor can be revealed from the inspection of emission lines, and in particular O I $6300 \AA$. For the present models $\mathrm{m} 12$ to $\mathrm{m} 25$, the oxygen mass increases with the ZAMS mass (it is $0.16,0.53,1.33$, and $2.33 M_{\odot}$ ), and this conspires to produce a greater $\mathrm{O}_{\mathrm{I}}$ line strength in more massive progenitors, which is apparent here in the bottom panel of Fig. 5.
Careful modeling is however needed to infer an accurate progenitor mass (Maguire et al. 2012; Jerkstrand et al. 2012, 2014).

Our results show that models from a $12,15,20$, and $25 M_{\odot}$ star on the main sequence can yield a type-II-P SN with a similar plateau duration and spectral evolution if they have the same H-rich envelope mass and ejecta kinetic energy. Differences arise primarily from offsets in progenitor radius (affecting the early-time brightness and color) and nucleosynthetic yields (affecting the lines from intermediate-mass elements at nebular times). Because of the unconstrained mass contribution from the He core, light-curve modeling cannot yield a robust inference of the ejecta mass, nor of the progenitor mass at the time of explosion. Furthermore, stars with different main sequence mass can die with the same $M_{\mathrm{H}, \mathrm{e}}$ (which is function of the uncertain cumulative mass loss), meaning that the constraint of $M_{\mathrm{H}, \mathrm{e}}$ from light-curve modeling cannot be uniquely connected to a $M_{\text {ZAMS }}$. Overall, there may well be a "RSG problem" but light-curve modeling will not solve it.

One may argue that this position is pessimistic. For example, from their set of progenitor stars and explosion models, Sukhbold et al. (2016) obtain a trend in asymptotic ejecta $E_{\text {kin }}$ and ${ }^{56} \mathrm{Ni}$ mass, and we may eventually have a robust prediction of these quantities from ab-initio 3D simulations of the explosion mechanism. We may also eventually have a robust theory for convection, overshoot, and RSG mass loss, with an accurate way to constrain core rotation. Similarly, we may eventually be able to perform multi-dimensional radiative transfer in nonLTE, thereby accounting accurately for the treatment of ejecta asymmetry, clumping, chemical segregation, and mixing. When this time comes, we will be able to constrain ejecta and progenitor masses with great confidence. We will have great accuracy in our results and therefore the degeneracies discussed in this paper will be lifted. However, this is not the present situation and even the state of the art remains limited by our understanding of each of the above building blocks.

\section{References}

Anderson, J. P., González-Gaitán, S., Hamuy, M., et al. 2014, ApJ, 786, 67 Arnett, W. D., Meakin, C., Viallet, M., et al. 2015, ApJ, 809, 30 Bartunov, O. S., \& Blinnikov, S. I. 1992, Sov. Astron. Lett., 18, 43 Beasor, E. R., \& Davies, B. 2016, MNRAS, 463, 1269

Bersten, M. C., Benvenuto, O., \& Hamuy, M. 2011, ApJ, 729, 61 Burrows, A., \& Goshy, J. 1993, ApJ, 416, L75

Chugai, N. N., \& Utrobin, V. P. 2000, A\&A, 354, 557

Crowther, P. A. 2007, ARA\&A, 45, 177

Dall'Ora, M., Botticella, M. T., Pumo, M. L., et al. 2014, ApJ, 787, 139

Davies, B., \& Beasor, E. R. 2018, MNRAS, 474, 2116

Davies, B., \& Dessart, L. 2019, MNRAS, 483, 887

de Jager, C., Nieuwenhuijzen, H., \& van der Hucht, K. A. 1988, A\&AS, 72, 259 Dessart, L., \& Hillier, D. J. 2011a, MNRAS, 410, 1739

Dessart, L., \& Hillier, D. J. 2011b, MNRAS, 415, 3497

Dessart, L., Livne, E., \& Waldman, R. 2010, MNRAS, 408, 827

Dessart, L., Hillier, D. J., Waldman, R., \& Livne, E. 2013, MNRAS, 433, 1745

Dessart, L., John Hillier, D., \& Audit, E. 2017, A\&A, 605, A83

Dessart, L., Hillier, D. J., \& Wilk, K. D. 2018, A\&A, 619, A30

Falk, S. W., \& Arnett, W. D. 1977, ApJS, 33, 515

Förster, F., Moriya, T. J., \& Maureira, J. C. 2018, Nat. Astron., 2, 574

Fraser, M., Maund, J. R., Smartt, S. J., et al. 2012, ApJ, 759, L13

Fraser, M., Maund, J. R., Smartt, S. J., et al. 2014, MNRAS, 439, L56

Glas, R., Just, O., Janka, H. T., \& Obergaulinger, M. 2019, ApJ, 873, 45

Grassberg, E. K., Imshennik, V. S., \& Nadyozhin, D. K. 1971, Astrophys. Space Sci., 10, 28

Hamuy, M. 2003, ApJ, 582, 905

Heger, A., Langer, N., \& Woosley, S. E. 2000, ApJ, 528, 368

Hillier, D. J., \& Dessart, L. 2012, MNRAS, 424, 252

Hirschi, R., Meynet, G., \& Maeder, A. 2004, A\&A, 425, 649

Jerkstrand, A., Fransson, C., Maguire, K., et al. 2012, A\&A, 546, A28

Jerkstrand, A., Smartt, S. J., Fraser, M., et al. 2014, MNRAS, 439, 3694

Kasen, D., \& Woosley, S. E. 2009, ApJ, 703, 2205 
L. Dessart and D. J. Hillier: Type II-P SNe and their progenitors

Langer, N., Hamann, W. R., \& Lennon, M. 1994, A\&A, 290, 819

Lentz, E. J., Bruenn, S. W., Hix, W. R., et al. 2015, ApJ, 807, L31

Leonard, D. C., Filippenko, A. V., Ganeshalingam, M., et al. 2006, Nature, 440, 505

Leonard, D. C., Pignata, G., \& Dessart, L. 2012, ATel, 4033

Lisakov, S. M., Dessart, L., Hillier, D. J., Waldman, R., \& Livne, E. 2017, MNRAS, 466, 34

Lisakov, S. M., Dessart, L., Hillier, D. J., Waldman, R., \& Livne, E. 2018 MNRAS, 473, 3863

Litvinova, I. Y., \& Nadezhin, D. K. 1985, Sov. Astron. Lett., 11, 145

Livne, E. 1993, ApJ, 412, 634

Maeder, A., \& Meynet, G. 1987, A\&A, 182, 243

Maguire, K., Jerkstrand, A., Smartt, S. J., et al. 2012, MNRAS, 420, 345

Maund, J. R., Smartt, S. J., \& Danziger, I. J. 2005, MNRAS, 364, L33

Maund, J. R., Fraser, M., Smartt, S. J., et al. 2013, MNRAS, 431, L102

Maund, J. R., Mattila, S., Ramirez-Ruiz, E., \& Eldridge, J. J. 2014, MNRAS, 438, 1577

Meynet, G., \& Maeder, A. 2000, A\&A, 361, 101

Meynet, G., Chomienne, V., Ekström, S., et al. 2015, A\&A, 575, A60

Moriya, T. J., Yoon, S.-C., Gräfener, G., \& Blinnikov, S. I. 2017, MNRAS, 469 , L108

Morozova, V., Piro, A. L., \& Valenti, S. 2017, ApJ, 838, 28

Morozova, V., Piro, A. L., \& Valenti, S. 2018, ApJ, 858, 15
Müller, B., Melson, T., Heger, A., \& Janka, H.-T. 2017, MNRAS, 472, 491 O'Connor, E. P., \& Couch, S. M. 2018, ApJ, 865, 81

O’Neill, D., Kotak, R., Fraser, M., et al. 2019, A\&A, 622, L1

Paxton, B., Bildsten, L., Dotter, A., et al. 2011, ApJS, 192, 3

Paxton, B., Cantiello, M., Arras, P., et al. 2013, ApJS, 208, 4

Paxton, B., Marchant, P., Schwab, J., et al. 2015, ApJS, 220, 15

Paxton, B., Schwab, J., Bauer, E. B., et al. 2018, ApJS, 234, 34

Popov, D. V. 1993, ApJ, 414, 712

Sanders, N. E., Soderberg, A. M., Gezari, S., et al. 2015, ApJ, 799, 208

Smartt, S. J. 2009, ARA\&A, 47, 63

Smartt, S. J., Maund, J. R., Hendry, M. A., et al. 2004, Science, 303, 499

Sukhbold, T., Ertl, T., Woosley, S. E., Brown, J. M., \& Janka, H.-T. 2016, ApJ, 821,38

Turatto, M., Mazzali, P. A., Young, T. R., et al. 1998, ApJ, 498, L129 Utrobin, V. P. 2007, A\&A, 461, 233

Van Dyk, S. D., Li, W., \& Filippenko, A. V. 2003, PASP, 115, 1289

Van Dyk, S. D., Davidge, T. J., Elias-Rosa, N., et al. 2012a, AJ, 143, 19

Van Dyk, S. D., Cenko, S. B., Poznanski, D., et al. 2012b, ApJ, 756, 131

Vartanyan, D., Burrows, A., Radice, D., Skinner, M. A., \& Dolence, J. 2019, MNRAS, 482, 351

Woosley, S. E. 1988, ApJ, 330, 218

Woosley, S. E., Heger, A., \& Weaver, T. A. 2002, Rev. Mod. Phys., 74, 1015

Yaron, O., Perley, D. A., Gal-Yam, A., et al. 2017, Nat. Phys., 13, 510 